\title{
Encuesta Nacional de Tendencias de Fitness en España para 2018 National Survey of Fitness Trends in Spain for 2018
}

\author{
*Oscar Luis Veiga Núñez, **Manel Valcarce Torrente, ***Adrian King Clavero, *Miguel Ángel de la Cámara Serrano \\ *Universidad Autónoma de Madrid (España), **Valgo Investiment (España), ***Mistral 2010 (España)
}

\begin{abstract}
Resumen. En 2016 se realizó la primera Encuesta Nacional sobre Tendencias en Fitness para 2017 en España reproduciendo la metodología de las encuestas anuales que ha venido realizando anualmente el Colegio Americano de Medicina del Deporte (American College of Sports Medicine, ACSM) sobre tendencias globales de fitness durante más de una década. El presente estudio presenta los resultados de la segunda Encuesta Nacional sobre Tendencias en Fitness para el año 2018, cuyo objetivo es identificar las principales tendencias y su relevancia específicamente en el sector del fitness español. La opinión de 637 profesionales del sector del fitness sobre 42 potenciales tendencias del sector para el año 2018 se recogió mediante un cuestionario online. Los resultados obtenidos se comparan y discuten con los resultados de la encuesta nacional y la encuesta internacional para el año 2017. Los resultados muestran que las tendencias en el sector del fitness en España para el año 2018 se corresponden en términos generales con las tendencias identificadas en la encuesta nacional de 2017 (17 tendencias coincidentes y tres nuevas), así como con las identificadas en la última encuesta internacional disponible (15 tendencias coincidentes). Las tendencias más relevantes en el contexto español son «personal formado, cualificado y experimentado», «entrenamiento funcional», «ejercicio y pérdida de peso», «entrenamiento interválico de alta intensidad», «entrenamiento personal» y «entrenamiento con el peso corporal»
\end{abstract}

Palabras clave: encuesta española fitness, tendencias fitness, industria fitness, encuesta ACSM

Abstract. In 2016 the first National Survey on Fitness Trend for 2017 in Spain was carried out reproducing the methodology of the annual surveys carried out by the American College of Sport Medicine (ACSM) about global fitness trends over more than a decade. This study shows the findings of the second National Survey of Fitness Trends for 2018, aimed to identify the main trends and their relevance in the Spanish fitness sector. The opinion of 637 professionals in the fitness sector about 42 potential trends in the sector for 2018 were gathered via an online questionnaire. Results are compared and discussed with the results of the national survey and the worldwide survey for 2017. In general terms, findings show that trends in the Spanish fitness sector for 2018 match with the trends identified in the national survey for 2017 (17 trends matched and three were new), as well as with the last worldwide survey (15 trends matched). The more relevant trends in the Spanish context are «educated, certified and experienced fitness professionals», «functional training», «exercise and weight loss», «high intensity intervallic training», "personal training» and «body weight training». Key words Spanish fitness survey, fitness trends, fitness industry, ACSM survey

\section{Introducción}

En el año 2016 se realizó la primera Encuesta Nacional sobre Tendencias en Fitness específicamente del sector español, reproduciendo los objetivos y la metodología de las encuestas internacionales sobre tendencias en fitness que el Colegio Americano de Medicina del Deporte(ACSM por sus siglas en inglés, American College of Sports Medicine) lleva realizando más de una década. Estas encuestas pretenden conocer de manera prospectiva cuáles serán, a nivel global, las principales tendencias del fitness. Los resultados de estas encuestas internacionales son difundidos anualmente a través de su revista oficial ACSM's Health and Fitness Journal (Thompson 2006, 2007, 2008, 2009, 2010, 2011, 2012, 2013, 2014, 2105 y 2016), mientras que la Encuesta Nacional de Tendencias en Fitness para 2017 ha sido difundida a través de la revista Apunts. Educación Física y Deportes en su número de julio de 2017 (Veiga, Valcarce \& King, 2017).

El objetivo principal, tanto de las encuestas internacionales como de su equivalente española, es proporcionar información sobre las tendencias que los profesionales del sector del fitness consideran que serán relevantes para el siguiente año y de este modo colaborar a que las organizaciones del sector puedan ajustar mejor su modelo de negocio y planificar acciones estratégicas sobre inversiones, oferta de productos y recursos humanos.

Asimismo, otro de los objetivos relevantes de estas encuestas es contribuir, a través de su repetición en el tiempo, a distinguir lo que pueden constituir verdaderas tendencias que emergen y se consolidan cada año de lo que pueden ser simples modas pasajeras. Para ello, el ACSM asume en sus encuestas la distinción entre tendencia (trend) y moda (fad) que se cita a continuación (Thompson, 2016, p.9)

Trend: «a general development or change in a situation or in the way that the people are behaving» (un desarrollo general o cambio en una situación o en la forma en que la gente se comporta»). Definición extraída según la fuente original del Cambridge Online Dictionary (s.f.)

Fecha recepción: 14-09-17. Fecha de aceptación: 30-11-17 Oscar Luis Veiga Núñez

oscar.veiga@uam.es
Fad: «a fashion that is taken up with entusiasm for a briefperiod of time» (una moda que es aceptada con entusiasmo por un breve periodo de tiempo). Definición extraída según la fuente original de Reference Online Dictionnary (s.f.)

Estas encuestas, tal y como señala el ACSM (Thompson, 2016), no abordan las tendencias relativas al equipamiento para gimnasios ni otros equipamientos de fitness para uso doméstico. Estas tratan de identificar tendencias que bien emergen en el sector o bien las que muestran consolidarse a través del tiempo en los cuatro ámbitos del fitness propuestos por el ACSM, esto es, el fitness comercial (entidades con ánimo de lucro), el fitness comunitario (entidades sin ánimo de lucro), el fitness médico (que incluyen programas de fitness terapéutico) y el fitness corporativo (programas de fitness en el ámbito laboral).

Las encuestas del ACSM se han estado llevando a cabo desde el año 2006 (para la identificación de las tendencias del 2007) y con la del año 2016 (para las tendencias del 2017) se han cumplido 11 ediciones de su realización. Esto ha proporcionado un amplio espectro de información que ha permitido observar la evolución de las diferentes tendencias identificadas y comprobar si se configuran como verdaderas tendencias con carácter duradero en el sector o si solo son «falsas tendencias», cuya característica principal es la de adquirir gran popularidad en un momento determinado para después caer rápidamente convirtiéndose, por tanto, en una moda.

Las encuestas del ACSM han mantenido en su ejecución una metodología estándar, si bien el número de países participantes en la misma ha variado mucho (véase Veiga, et al., 2017) y España solo aparece como participante en la encuesta del 2015 (Thompson 2014). La Encuesta Nacional de Tendencias en Fitness para el año 2017 permitió comprobar que muchas de las tendencias identificadas a nivel global en las encuestas del ACSM son también tendencias en el sector del fitness español, a la vez que ha servido para identificar algunas tendencias que parecen ser particulares de nuestro país. Asimismo, ha permitido comprobar que la relevancia otorgada por las encuestas internacionales y española a algunas tendencias comunes es considerablemente diferente, y también que ciertas tendencias relevantes en el contexto internacional aún no se han manifestado como tal en nuestro país. Atendiendo a las particularidades dadas en el contexto español, parece relevante y de interés poder continuar con la realización de una encuesta nacional que 
permita orientar específicamente a las organizaciones y a los profesionales del sector en nuestro país.

Por tanto, el objetivo del presente estudio ha sido analizar las tendencias del fitness en el contexto español para el año 2018 a través de encuesta a profesionales del sector, tomando como base la metodología propuesta por el ACSM para el desarrollo de sus encuestas internacionales sobre tendencias en el sector del fitness, y comparar los resultados obtenidos con la encuesta nacional e internacional del año 2017.

\section{Material y métodos}

Para la selección de tendencias de esta encuesta se incluyeron las 30 primeras tendencias identificadas para el año 2017 por el orden de puntuación obtenida. Además, se incorporaron dos tendencias que aparecieron en el top 20 de la encuesta internacional para el año 2017 pero que no fueron recogidas dentro de las 30 tendencias anteriores (la iniciativa de la ACSM «Ejercicio es Medicina ${ }^{\circledR}$ » $\mathrm{y}$ «clases colectivas»). Asimismo, se consideró conveniente separar en dos la tendencia relativa a «corrección postural y prevención y recuperación de lesiones» (dividiéndola en «corrección postural y fitness postural» y «prevención y readaptación funcional de lesiones») pues esa forma de presentación agrupada podría estar ocultando cuál de las dos es en realidad verdadera tendencia, como así lo ponen de manifiesto los resultados obtenidos. Por tanto, en la presente encuesta se presentaron como tendencias diferenciadas. Finalmente, los autores del estudio propusieron un total de 9 tendencias adicionales a incorporar en la encuesta para completar un total de 42 potenciales tendencias que fueron incorporadas al estudio al igual que en la encuesta internacional de 2017.

Para valorar la relevancia de las tendencias potenciales seutilizóuna escala tipo Likert de 1 (menor probabilidad de ser una tendencia) a 10 (mayor probabilidad de ser una tendencia) reproduciendo el procedimiento de valoración de las encuestas del ACSM. Se proporcionó además un espacio para que los encuestados pudieran incluir otras tendencias potenciales y que no estuviesen incluidas en el cuestionario. Asimismo, se recogió información sobre el perfil de los encuestados incluyendo su sexo, edad, comunidad autónoma de residencia, número de años de experiencia en el sector, ámbito del fitness en el que trabaja, su ocupación y situación laboral, así como su lugar principal de trabajo entre otras.

La encuesta se envió electrónicamente a una lista de profesionales del sector del fitness $(n=12.531)$ utilizando la plataforma online de encuestas Survio. Esta lista de profesionales incluía tanto profesionales del sector privado de diferentes tipos de centros de fitness (premium, medio, low cost y estudios/boutiques) como del sector público (centros de gestión pública y de gestión privada), además de incluir a profesionales autónomos. La encuesta se mantuvo abierta y pudo ser respondida por un periodo de 4 semanas del 2 al 30 junio de 2017. Tras el envío inicial de la encuesta se realizaron dos recordatorios por email a los potenciales respondedores. Finalmente, un total de 637 sujetos respondieron a la encuesta (ratio de respuesta de 5,1\%.), obteniéndose respuestas provenientes de todas las comunidades autónomas españolas, así como de Ceuta y Melilla. Si bien se trata de una muestra incidental sin selección aleatoria, la estimación de error muestral bajo supuestos de muestreo aleatorio simple, población infinita, nivel de confianza del 95\% y $\mathrm{P}=\mathrm{Q}$ sería de $\pm 3.9 \%$.

Para identificar las tendencias más relevantes, se calculó la media de las puntaciones obtenidas por cada tendencia en la encuesta, ordenándose de mayor a menor. Las 20 tendencias con mayor puntuación fueron seleccionadas para ser presentadas en este informe, siguiendo la pauta utilizada en las encuestas internacionales del ACSM (que denomina a esta relación «top 20»). Para el presente estudio se ha considerado discutir los resultados obtenidos contrastándolos tanto con la Encuesta Nacional de Tendencias en Fitness para 2017 realizada por primera vez en España el año pasado, así como con la última encuesta internacional disponible, es decir, la realizada por el ACSM para el año 2017.

\section{Resultados}

La Tabla 1 presenta las principales características de los encuestados. Un 29,8\% de la muestra fueron mujeres y ésta cubrió un amplio rango de edades, encontrándose el grupo mayoritario de edad entre los 22 y los 34 años (42,2\%). Más de la mitad de los encuestados manifestó tener una experiencia profesional en el sector por encima de los 10 años $(55,2 \%)$ y desarrollar su labor profesional dentro del sector del fitness comercial (52,9\%). En cuanto a la situación laboral, la mayor parte de los encuestados trabajaba en el sector del fitness como ocupación principal (83,2\%) y un $67,2 \%$ trabajaba a tiempo completo mientras que el 20,5\% lo hacía a tiempo parcial, teniendo el resto otras situaciones (Tabla 1). Por otro lado, el lugar de trabajo de los encuestados fue mayoritariamente centros privados (52,5\%) mientras que aproximadamente el 30\% lo hacía en centros públicos y el resto en espacios al aire libre, a domicilio o en estudios o boutiques (Tabla 1).

\begin{tabular}{lc} 
Tabla 1 & \\
Características descriptivas de la muestra en porcentajes & \\
\hline Sexo & \\
Mujer & 29,8 \\
Hombre & 70,2 \\
Edad & \\
Menor 21 años & 0,8 \\
De 22 a 34 años & 42,2 \\
De 35 a 44 años & 37,2 \\
De 45 a 54 años & 14,4 \\
Mayor 55 años & 5,3 \\
Años de experiencia en el sector & \\
Menos de 1 año & 3,1 \\
De 1 a 3 años & 8,8 \\
De 3 a 5 años & 12,2 \\
De 5 a 7 años & 9,9 \\
De 7 a 9 años & 10,7 \\
De 10 a 20 años & 40,0 \\
Más de 20 años & 15,2 \\
Lugar de trabajo & \\
Centro privado & 52,5 \\
Centro público & 29,5 \\
Estudio o boutique & 8,0 \\
Espacios al aire libre & 11,5 \\
A domicilio & 9,6 \\
Otro & 7,4 \\
Sector fitness en el que trabaja & \\
Fitness comercial & 52,9 \\
Fitness comunitario & 12,7 \\
Fitness corporativo & 17,1 \\
Fitness médico & 10,8 \\
Situación laboral en sector fitness & \\
Ocupación principal & 83,2 \\
Segunda ocupación & 10,2 \\
Tercera ocupación o más & 6,6 \\
Trabajo a tiempo completo & 67,2 \\
Trabajo a tiempo parcial sin otro trabajo & 8,6 \\
Trabajo a tiempo parcial con otro trabajo \\
He trabajado en el sector pero he cambiado \\
No trabajo actualmente en el sector, estoy en paro \\
Otros & 11,9 \\
$\quad$ & 6,0 \\
& 1,7 \\
& 6,0 \\
\hline
\end{tabular}

Tabla 2

Ocupaciones y nivel de ingresos de los encuestados en porcentaje.

Propietario centro/Enpesa

Gerente

Director técnico

Coordinador/a

Monitor/a multidisciplinar

Monitor/a de sala

Monitor/a de clases colectivas

(anterieno completo)

Entrenador/a personal (a tiempo parcial)

Profesor/a

Maestro/a

Profesional de la salud (médico, fisioterapeuta, terapeuta ocupacional...)

Estudiante graduado/a

Estudiante no graduado

Ninguno. No estoy trabajando en el sector del fitness

Otros

Ingresos brutos anuales

Menos de $10.000 €$

De 10.000 a $14.999 €$

De 15.000 a $19.999 €$

De 20.000 a $24.999 €$

De 25.000 a $29.999 €$

De 30.000 a $34.999 €$

De 35.000 a $39.999 €$

De 40.000 a $44.999 €$

De 45.000 a $49.999 €$

Más de $50.000 €$ 
Tabla 3

Ranking de tendencias de fitness en las dos encuestas nacionales (años 2017 y 2018) y en la última encuesta internacional (año 2017)

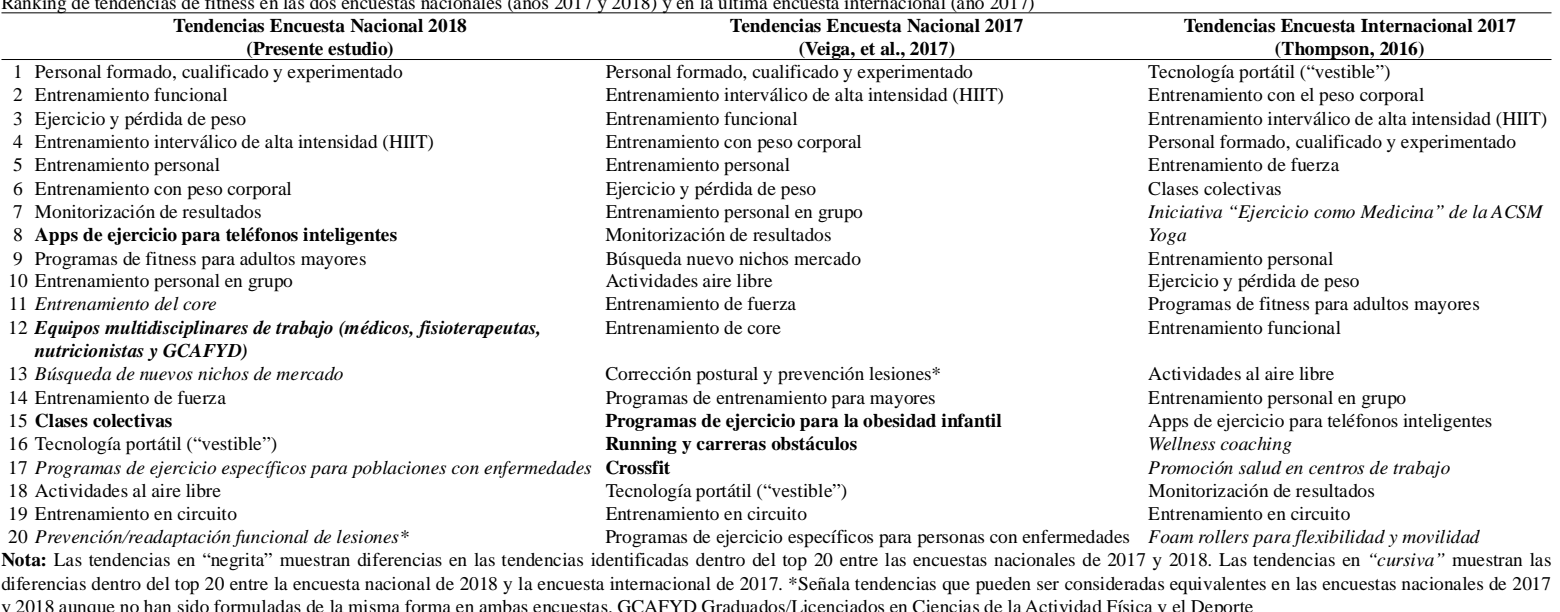

y 2018 aunque no han sido formuladas de la misma forma en ambas encuestas. GCAFYD Graduados/Licenciados en Ciencias de la Actividad Física y el Deporte

Las ocupaciones de los encuestados fueron diversas y son recogidas en la Tabla 2, destacando que el 36,7\% eran monitores, el 26,8\% entrenadores personales a tiempo parcial o completo y el $24,2 \%$ gerentes. En relación a las retribuciones por el trabajo desempeñado, cabe destacar que aproximadamente un $60 \%$ de los encuestados obtuvieron unos ingresos por debajo de los 25.000 • brutos anuales y un tercio (32,9\%) por debajo de 15.000 .

Para el análisis e interpretación de los resultados obtenidos en la encuesta, las tendencias han sido ordenadas de mayor a menor puntuación según la percepción de los encuestados sobre su probabilidad de ser una tendencia dentro del ámbito del fitness en el año 2018. Como se ha señalado en el apartado de métodos, en este trabajo se presenta el top 20 del ranking según la puntuación obtenida tal y como se realiza en los estudios publicados por el ACSM. Asimismo, se presentan las tendencias identificadas en el top 20 de la encuesta española de 2017 (Veiga, et al., 2017) y en la encuesta internacional de 2017 (Thomas, 2016) con objeto de contrastar los resultados obtenidos en las tres encuestas (Tabla 3).

Como puede observarse, 17 de las tendencias identificadas en el top 20 de la encuesta española de 2018 corresponden con tendencias dentro del top 20 de la encuesta de 2017 y solo tres corresponden a tendencias diferentes. Estas fueron: «apps para teléfonos inteligentes», «equipos multidisciplinares de trabajo» $\mathrm{y}$ «clases colectivas». Por otro lado, puede reseñarse que la tendencia que ocupa el primer puesto en la presente encuesta es «personal formado, cualificado y experimentado» y que esta ocupó asimismo el primer puesto en la encuesta nacional de 2017. Del mismo modo, las siguientes cinco posiciones del ranking son ocupadas por las tendencias «entrenamiento funcional», «ejercicio y pérdida de peso», «entrenamiento interválico de alta intensidad», «entrenamiento personal» y «entrenamiento con peso corporal», pudiéndose observar que las primeras posiciones en la encuesta nacional de 2018 y de 2017 están ocupadas por las mismas tendencias, si bien variando ligeramente sus posiciones.

Por otro lado, del top 20 de la encuesta nacional del 2018, 15 tendencias corresponden con el top 20 de la última encuesta internacional, con solo cinco tendencias diferentes (Tabla 3). Estas fueron: «entrenamiento del core», «equipos multidisciplinares de trabajo», «búsqueda de nuevos nichos de mercado», «programas de ejercicio específicos para poblaciones con enfermedades» $\mathrm{y}$ «prevención y readaptación de lesiones». En cuanto a las tendencias que ocupan los primeros puestos en la encuesta nacional de 2018 solo tres coinciden con las primeras tendencias de la encuesta internacional de 2017 («personal formado cualificado y experimentado, «entrenamiento interválico de alta intensidad» $\mathrm{y}$ «entrenamiento con peso corporal»).

\section{Discusión}

El objetivo de este estudio ha sido identificar las tendencias de fitness en España para el año 2018 según la opinión de los profesionales del sector, así como comparar los resultados obtenidos con las tendencias identificadas por las encuestas nacional e internacional del año 2017. Para ello se ha realizado una encuesta online que fue enviada a un amplio número de profesionales del sector del fitness en España. Aunque el procedimiento de encuesta realizado no permite establecer la representatividad de la muestra ni de los resultados, la muestra ha abarcado a profesionales procedentes de todas las comunidades autónomas españolas, que trabajan tanto en el sector privado como público, incluyendo un amplio rango de edades y ocupaciones, con más de un 80\% con dedicación principal al sector de fitness, casi un 70\% de empleados a tiempo completo y una representación femenina del 30\%.

De forma general puede señalarse, respecto a los resultados obtenidos para las tendencias correspondientes al año 2018, que mayoritariamente corresponden con las tendencias identificadas para el año 2017 tanto en la encuesta nacional (17 tendencias comunes de 20) como para la encuesta internacional (15 de 20). Por otro lado, puede señalarse que las posiciones ocupadas por las tendencias en las encuestas nacionales de 2017 y 2018 son muy similares, solo apreciándose diferencias notables para las tendencias «actividades al aire libre» y «corrección postural y prevención/recuperación de lesiones». Asimismo, se aprecian diferencias moderadas en las tendencias de «búsqueda de nuevos nichos de mercado» y «programas de entrenamiento para mayores». Por otro lado, es destacable que la tendencia «personal formado, cualificado y experimentado» ocupa el $\mathrm{n}^{\circ} 1$ del top en ambas encuestas, y que las seis primeras tendencias de ambas encuestas son las mismas, aunque en diferente orden. En resumen, ambas encuestas muestran resultados muy similares, lo que significa que en términos generales las tendencias identificadas en 2017 en el contexto español se mantienen en el tiempo un año después.

Sin embargo, la diferencia en la posición ocupada por las diversas tendencias resulta más notable cuando se comparan con las tendencias internacionales de 2017, identificándose hasta cuatro tendencias que muestran una diferencia de más de 10 posiciones en su situación en el ranking y otras cuatro que muestran una diferencia de cinco posiciones o más. Cabe destacar la diferencia en las tendencias que ocupan las posiciones 1 y 5 en la encuesta internacional de 2017 («tecnología portátil vestible» $\mathrm{y}$ «entrenamiento de fuerza») que, sin embargo, en la encuesta española de 2018 ocupan las posiciones 16 y 14 respectivamente.

A continuación, se comentan y discuten una por una las tendencias 
dentro del top 20 de la encuesta española para el año 2018, describiéndolas brevemente en base a su conceptualización en los estudios internacionales del ACSM, y contrastando los resultados obtenidos por cada tendencia en las encuestas tanto española como internacional para el año 2017.

1. Profesionales formados, cualificados y con experiencia. Esta tendencia vuelve a ocupar el no 1 del top 20 para el año 2018, como ya lo hizo en el estudio de 2017. Esta tendencia hace referencia a la necesidad detectada en el sector del fitness de contar con profesionales debidamente formados, con adecuada cualificación profesional y experiencia. En la encuesta internacional de 2017 ocupó la $4^{\circ}$ posición ubicándose, por tanto, también en las primeras posiciones. Puede señalarse aquí que esta tendencia ha ocupado la primera posición durante 6 años en las encuestas internacionales y siempre ha estado situada entre los 5 primeros puestos en los 11 años que esas encuestas llevan realizándose.

2. Entrenamiento funcional. El entrenamiento funcional se define en las encuestas del ACSM como el uso de entrenamiento de fuerza para mejorar el equilibrio, la coordinación, la fuerza, la potencia y la resistencia que permite a la persona mejorar su capacidad para llevar a cabo las actividades de la vida diaria. El entrenamiento funcional ha ocupado posiciones intermedias en las encuestas internacionales, incluida la última de 2017 donde ocupa el puesto ${ }^{\circ} 12$. Por el contrario, en el caso español, en la encuesta para el 2017 ocupó una posición muy destacada (posición $n^{\circ}$ 3) y este año ha escalado una posición más, posicionándose en la encuesta para 2018 en el puesto $n^{\circ} 2$. Esta es una de las tendencias donde las diferencias son más notables entre los resultados arrojados por las encuestas españolas y la encuesta internacional de 2017 con una diferencia entorno a 10 posiciones en el ranking y, en general, con la posición que esta tendencia ha ocupado en el histórico de encuestas del ACSM.

3. Ejercicio y pérdida de peso. Los programas que proponen una combinación de ejercicio y restricción calórica para la pérdida de peso se sitúan este año como la $3^{\mathrm{a}}$ tendencia en el sector del fitness español, escalando 3 posiciones respecto a la encuesta de 2017, donde se situó en el puesto $\mathrm{n}^{\circ} 6 \mathrm{y}$, por tanto, incrementando la importancia que parecen concederle los profesionales del sector. En la encuesta internacional de 2017 ocupó una posición mucho menos relevante que en la encuesta española de 2018, situándose en el puesto 10. Bien es cierto que esta ha sido una tendencia que ha aparecido dentro del top 20 en todas las encuestas internacionales desde 2007, en general en puestos intermedios, aunque entre los años 2011 a 2013 llegó a alcanzar los puestos 4 y 5 , es decir, parecidos a los ocupados actualmente en las encuestas españolas.

4. Entrenamiento interválico de alta intensidad (HIIT). El HIIT (High Intensity Interval Training) hace referencia a un entrenamiento que incluye intervalos de ejercicio de alta intensidad seguidos por cortos periodos de descanso y que habitualmente supone menos de $30 \mathrm{minu}-$ tos de trabajo total. Esta tendencia pierde posiciones respecto a la encuesta nacional de 2017, pasando del puesto 2 al 4, si bien sigue siendo una de las tendencias principales de fitness en España. En la encuesta internacional de 2017 ocupa también una posición muy relevante (puesto $\mathrm{n}^{\circ} 3$ ). Esta es una tendencia que apareció por primera vez en los estudios internacionales en 2014 y que desde entonces se ha mantenido entre los primeros puestos, lo que es coherente con lo que se ha observado en las encuestas españolas.

5. Entrenamiento personal. El entrenamiento personal se mantiene dentro de las tendencias principales del sector del fitness ocupando el puesto $n^{\circ}$ 5, la misma posición que en el año 2017. Esta tendencia hace referencia al entrenamiento dirigido para un único cliente por un profesional. Su relevancia en el ámbito español parece sensiblemente mayor que la que presenta en la actualidad en el ámbito internacional donde ocupa el puesto $n^{\circ} 9$ según la encuesta de 2017, si bien en el pasado llegó a ocupar el puesto $\mathrm{n}^{\circ} 3$ en los años 2008 y 2009.

6. Entrenamiento con peso corporal. El entrenamiento con peso corporal pierde dos posiciones en relación a la encuesta nacional de 2017 pasando del puesto $\mathrm{n}^{\circ} 4 \mathrm{al} \mathrm{n}^{\circ} 6$. Esta tendencia hace referencia a paquetes de entrenamiento ofertados en los centros de fitness realizados con un mínimo equipamiento y usando la carga del peso del cuerpo como principal forma de producir sobrecarga muscular y acondicionamiento físico. Esta tendencia, que apareció en los estudios internacionales en 2013, ha ocupado siempre posiciones muy relevantes en las encuestas del ACSM con puestos entre el $1^{\circ}$ y el $3^{\circ}$, ocupando el $2^{\circ}$ puesto en la última encuesta de 2017. Estos resultados en el histórico de encuestas internacionales parecen indicar una relevancia ligeramente mayor de esta tendencia en el contexto internacional que en el español.

7. Monitorización de resultados. La monitorización de resultados ha pasado del puesto 8 en 2017 al puesto 7 en 2018 en las encuestas españolas, mientras que en la encuesta internacional de 2017 ocupa una posición mucho menos relevante (puesto 18) indicando que es una tendencia mucho menos relevante en la actualidad en el ámbito internacional. Por otro lado, aunque dicha tendencia ha aparecido dentro del top 20 en todas las encuestas internacionales realizadas nunca ha ocupado posiciones por debajo del puesto 10 .

8. Apps de ejercicio para teléfonos inteligentes. Aparece este año con fuerza dentro de las 20 tendencias más relevantes en la encuesta española, posicionándose dentro de las 10 tendencias más relevantes, mientras que el año pasado no consiguió entrar dentro de ese top 20 (ocupó el puesto ${ }^{\circ} 21$ ). Las apps relacionadas con el ejercicio, el fitness y el deporte han adquirido mucha popularidad en los últimos años y es previsible que la sigan adquiriendo. Como ejemplos de este tipo de apps, podemos citar algunas de las más populares como Nike Training Club, Cyclemeter by Advio, MyFitnessPal, Endomondo Pro, Reebook Fitness, Runtastic. Estas apps permiten realizar el seguimiento de la actividad realizada a lo largo del tiempo y obtener feed-back sobre el progreso. Bien es cierto que una de las limitaciones de estas apps es que la validez de los datos aportados por estos dispositivos es aún cuestionable. En todo caso, cabe esperar que su precisión y validez sigan mejorando y sean un elemento que contribuya a la monitorización de los programas de ejercicio en el futuro.

9. Programas de entrenamiento para mayores. Los programas de acondicionamiento físico orientados a personas mayores han escalado 5 posiciones pasando del puesto $\mathrm{n}^{\circ} 14$ en la encuesta de 2017 al puesto $n^{\circ} 9$ en la de 2018, lo que parece indicar la emergencia de esta tendencia en el sector. Este resultado parece congruente con el crecimiento acelerado de esta población en los países desarrollados, incluido España. Esta tendencia hace referencia a programas de ejercicios supervisados dirigidos a personas por encima de la edad de jubilación, adecuados a las características de esta población y que resulten seguros. Muchos sectores productivos han identificado lo que se denomina el «silver market phenomenon» (Kohlbacher y Hertastt, 2008), es decir, un nicho de demanda específico asociado a un sector de la población en crecimiento exponencial, con necesidades específicas, mucha disponibilidad de tiempo libre y una capacidad adquisitiva apreciable en los países desarrollados. Esta tendencia en la encuesta internacional de 2017 ocupa una posición parecida a la de las encuestas españolas (puesto $n^{\circ} 11$ ) que, sin embargo, es la posición más baja ocupada por dicha tendencia en el histórico de encuestas internacionales, donde ha llegado a ocupar la posición n 2 en 2007 y 2011 y n 3 en 2013. Los datos históricos de las encuestas internacionales parecen señalar una disminución de su importancia a nivel global mientras que en España los resultados sugieren que su importancia puede estar aumentando.

10. Entrenamiento personal en grupo. El entrenamiento personal en grupo muestra para el año 2018 una ligera disminución de la relevancia que los profesionales le otorgan pasando del puesto 7 al 10. El entrenamiento personal en grupo sigue el modelo de entrenamiento personal individual buscando una atención personalizada del profesional al cliente, pero en este caso en pequeños grupos usualmente de entre 2 y 4 personas, permitiendo de esta forma recibir una atención personalizada pero con un coste por el servicio significativamente menor. El entrenamiento personal en grupo alcanzó las posiciones más relevantes en las encuestas internaciones en los años 2012 a 2015 (con puestos entre el $8^{\circ}$ y $10^{\circ}$ ), lo cual puede considerarse como una respues- 
ta del sector a la crisis económica en la prestación de servicios de entrenamiento personal. En la encuesta internacional del 2017 su posición es más modesta ocupando la posición n n 14, lo que puede también ser un reflejo en el sector del fitness de la recuperación económica a nivel global.

11. Entrenamiento del core. El entrenamiento del core se orienta el fortalecimiento y acondicionamiento de los músculos estabilizadores del abdomen, el tórax y la espalda, para mejorar la estabilización del tronco y permitir la transferencia de fuerza a las extremidades, capacitando así a los individuos para hacer frente de mejor manera tanto a actividades de la vida diaria como a actividades deportivas. Este tipo específico de entrenamiento se manifiesta como una tendencia relevante en el sector del fitness español, mientras que en el ámbito internacional ha desaparecido del top 20 en el año 2017 después de un progresivo declive en la importancia otorgada por los profesionales. Así, esta tendencia ocupó el $5^{\circ}$ puesto en las encuestas desde 2007 a 2011, para ir descendiendo progresivamente de posición a partir de 2011 hasta alcanzar el puesto 19 en la encuesta de 2016 y salir finalmente del top 20 en la última encuesta del 2017. Queda por ver qué pasará en las encuestas internacionales de los próximos años y si queda fuera definitivamente del top 20 o recobra importancia y vuelve a entrar. También será de interés observar si en el sector del fitness español se reproducirá la progresión a la baja en su relevancia en los próximos años.

12. Equipos multidisciplinares de trabajo (médicos, fisioterapeutas, nutricionistas, psicólogos y GCAFYD). Esta es una de las tendencias propuestas por los autores del estudio que no posee un equivalente en los estudios internacionales. Hace referencia al trabajo conjunto de diferentes profesionales, desarrollado de forma coordinada, y con el objetivo de mejorar la salud de aquellas personas que pueden obtener beneficios óptimos de una labor integrada y mutidisciplinar. Esta tendencia ha sido propuesta por primera vez para la encuesta española de este año por lo que tampoco resulta posible la comparación con la encuesta del año pasado, si bien, resulta reseñable que entra en el top 20 con una posición destacable (puesto $\mathrm{n}^{\circ} 12$ ).

13. Búsqueda de nuevos nichos de mercado. La tendencia de búsqueda de nuevos nichos de mercado descendió del puesto 9 en 2017 al puesto 13 en 2018 en las encuestas nacionales. Esta tendencia hace referencia a la búsqueda de nuevos grupos de clientes que puedan ser atraídos al sector de fitness mediante la creación de productos y servicios orientados a cubrir sus necesidades específicas. Esta tendencia, sin embargo, es una de las que no aparece dentro del top 20 en la encuesta internacional de 2017 del ACSM. Esta tendencia ha estado presente en el top 20 de las encuestas internacionales desde 2007 a 2013 (con excepción del año 2010) pero a partir de 2014 no aparece como una tendencia relevante en el ámbito internacional, lo cual constituye una de las diferencias más remarcables entre las tendencias internacionales y las tendencias en el ámbito nacional.

14. Entrenamiento de fuerza. El entrenamiento de fuerza pasa del puesto 11 en 2017 al puesto 14 en 2018. Esta tendencia hace referencia al entrenamiento específicamente orientado al desarrollo de la fuerza y masa muscular y que involucra normalmente el uso de máquinas contraresistencia o el uso de cargas libres para producir los estímulos de entrenamiento necesarios al sistema muscular. Esta tendencia ocupa una posición mucho más relevante en la encuesta internacional de 2017 (puesto ${ }^{\circ}$ 5) y de hecho es una de las tendencias que siempre ha estado entre los 5 primeros puestos en todas las encuestas internacionales con excepción del año 2007 que ocupó el nº 6 . Resulta, por tanto, reseñable las diferencias en cuanto a su posición entre las encuestas nacionales e internacionales.

15. Clases colectivas (Group training). Esta tendencia se incorpora dentro del top 20 en la encuesta nacional de 2018 después de su aparición por primera vez en la encuesta internacional de 2017, motivo por el que fue seleccionada dentro de las 42 tendencias potenciales de la encuesta. Esta tendencia hace referencia a actividades donde los instructores enseñan, dirigen y motivan a grupos grandes de individuos de diferentes niveles de condición física para conseguir sus objetivos de manera eficaz. Esta tendencia ha sido considerada como una tendencia potencial en las 11 encuestas intencionales realizadas hasta el momento por el ACSM, pero solo en la encuesta de 2017 ha emergido como una tendencia relevante ocupando además el puesto $\mathrm{n}^{\circ} 6$ del ranking. $\mathrm{Al}$ incluirse en la presente encuesta española, esta también ha conseguido situarse entre las 20 primeras tendencias, si bien en una posición no tan destacada.

16. Tecnología portátil vestible («wereables»). Esta tendencia gana puestos en la encuesta de 2018 respecto a la de 2017 pasando del puesto 18 al puesto 16, pero no muestra en el contexto nacional la relevancia que muestra en el contexto internacional, donde ha ocupado la primera posición tanto en la encuesta de 2017 como en la de 2016 donde fue incluida por primera vez. Esta tendencia hace referencia a toda aquella tecnología portátil y «vestible» que puede ayudar a mejorar o monitorizar el entrenamiento. Esto incluye pulsómetros, relojes inteligentes y dispositivos de monitorización de actividad y fitness (como Misfit, Garmin; Jawbone, Fitbit, etc.). En el futuro, según se señala en el último estudio del ACSM (Thompson, 2016), posiblemente se extenderá a los tejidos inteligentes e interactivos que se están desarrollando y optimizando en la actualidad. Algunos datos relevantes sobre la proyección de crecimiento del mercado de «wereables» son señalados en la presentación de resultados de la última encuesta del ACSM. Por ejemplo, se señala que están previstas unas ventas de iWatch de Apple superiores a 485 millones de dispositivos y la previsión de negocio para 2017 de los dispositivos tipo «smart glasses» se situó en torno a los 1.500 millones de dólares y para la ropa inteligente y los tejidos interactivos en torno a 2.600 millones de dólares. El crecimiento de esta tendencia parece asegurado en el futuro, pero resulta poco predecible cuál será su desarrollo y cómo afectará al sector del fitness (Thompson, 2016).

17. Programas de ejercicio específicos para personas con enfermedades. Esta tendencia hace referencia al diseño y desarrollo de programas de entrenamiento específicos para personas con enfermedades (cardiovasculares, diabetes, cáncer, osteoporosis, demencias, etc.). Su posición mejora 3 puestos en relación a la encuesta de 2017 donde ocupó el último lugar del top 20, lo que parece indicar un ligero incremento de su relevancia. Esta tendencia fue una de las 4 tendencias que emergió en la encuesta nacional de 2017 como específica del sector del fitness español y que no fue propuesta ni seleccionada a partir de las encuestas previas del ACSM. No existe, por tanto, la tendencia equivalente dentro de las encuestas internacionales, por lo que no es posible valorarla en relación a éstas. Sin embargo, en la encuesta internacional de 2017 aparece por primera vez la tendencia «Ejercicio es Medicina ${ }^{\circledR}$ » que es una iniciativa internacional del ACSM estimulando a los médicos de atención primaria y otros profesionales de la salud a incluir la actividad física en el diseño de sus planes de tratamiento y a derivar a sus pacientes hacia profesionales especialistas en ejercicio. Si bien estas tendencias no son directamente comparables, parece obvio que están relacionadas pues ambas se orientan a que la actividad física constituya un elemento fundamental del tratamiento de aquellas enfermedades y patologías en las que existe evidencia sobre su eficacia terapéutica. Hay que señalar que la iniciativa «Ejercicio es Medicina ${ }^{\circledR} »$ sí se ha incorporado dentro de las 42 tendencias potenciales en la encuesta española de 2018, quedando ubicada en el puesto 32 del ranking y, por tanto, no forma parte del top 20 de tendencias. Lo cierto es que la incorporación de España a esta iniciativa internacional es bastante reciente (año 2014) y hasta el momento ha tenido una difusión muy limitada en nuestro país, tanto entre los profesionales de la salud como de los profesionales del fitness, por lo que en realidad su filosofía y contenidos son poco conocidos.

18. Actividades al aire libre. Las actividades al aire libre bajan de posición en el ranking de 2018 pasando del puesto 10 en el 2017 al puesto 18 en la presente encuesta, lo cual supone un descenso significativo. Este tipo de actividades incluyen actividades como el senderismo, escala deportiva, la canoa, el kayak, así como juegos y deportes al aire libre, y también aquellas actividades de acondicionamiento físico que los profesionales ofrecen a sus clientes al aire libre. Esta tendencia también 
aparece dentro del top 20 de la encuesta internacional de 2017 ocupando el puesto $n^{\circ} 13$, posición muy parecida a los puestos ocupados otros años desde que apareció por primera vez dentro del top 20 en 2012.

19. Entrenamiento en circuito. El entrenamiento en circuito hace referencia usualmente a un entrenamiento donde se realizan de 6 a 10 ejercicios en una secuencia preestablecida, de modo que cada ejercicio se realiza bien un número determinado de veces o bien por un periodo de tiempo determinado, realizando después un breve descanso antes de pasar a otro ejercicio. Esta tendencia ocupa la misma posición en la encuesta de 2018 y de 2017 ubicándose en el penúltimo puesto del ranking, mientras que en la encuesta internacional de 2017 ocupó la posición $n^{\circ} 13$, similar a la que ha ocupado otros años desde que apareció dentro del top 20 en el año 2012. Parece por tanto una tendencia bastante estable dentro del conjunto en la importancia concedida por los profesionales del sector.

20. Prevención/readaptación funcional de lesiones. Esta tendencia hace referencia a programas de ejercicio orientados tanto a la prevención de lesiones como a la readaptación funcional y el re-entrenamiento una vez sufrida una lesión y pasada la fase de recuperación fisioterapéutica. El objetivo de estos programas es conseguir poner a la persona que ha sufrido la lesión en una situación de acondicionamiento físico adecuado para volver a rendir físicamente y a la vez tratar de evitar reincidencias en la lesión. Esta es una tendencia específicamente española con la que no es posible la comparación con la encuesta internacional de 2017. Por otro lado, la comparación con los resultados de la encuesta española de 2017 tampoco es directa pues esta tendencia se contempló en la encuesta anterior de forma combinada con la corrección postural, proponiéndose este año, sin embargo, de forma separada como se ha explicado en al apartado de métodos. En la encuesta de 2017 la tendencia que se denominó «corrección postural, prevención y recuperación de lesiones» ocupó un puesto intermedio del ranking (puesto 13) mientras que en la encuesta de este año la tendencia analizada ocupa laúltima posición del ranking, mientras que la tendencia de «corrección postural y fitness postural» ha quedado fuera del top 20 ocupando la posición 24. Valorado en conjunto, puede interpretarse que la relevancia de estos programas de entrenamiento parece haber perdido importancia respecto a 2017.

\section{¿Qué queda fuera en la encuesta de 2018?}

En relación a la encuesta de 2017 varias tendencias han salido del top 20 en el año 2018. En primer lugar, los «programas de ejercicio para la obesidad infantil», que este año ha estado cerca de entrar dentro del top 20 (puesto 22) pero que ha descendido 7 puestos desde el $n^{\circ} 15$ que ocupó en 2017, lo que supone una disminución importante de la relevancia concedida a esta tendencia. Las tendencias de «crossfit» y «running y carreras de obstáculos», que fueron dos tendencias propuestas específicamente en el estudio español de 2017, salen también fuera del top 20 . El «crossfit» pasa del puesto 17 al puesto 26, mientras que el «running y las carreras de obstáculos» desciende muy pronunciadamente del puesto 16 al puesto 41 en 2018. En el caso de estas dos últimas tendencias, que han salido del top 20 con una diminución tan pronunciada en su valoración en un año, puede interpretarse que se han tratado de actividades que simplemente se pusieron de moda y no representar verdaderas tendencias.

\section{¿Qué se incorpora en la encuesta de 2018?}

Asimismo, existen 3 tendencias que se incorporan en 2018 al top 20 de la encuesta relevando a las 3 tendencias que salen. La primera de ellas es «apps de ejercicio para teléfonos inteligentes». Esta tendencia, que no entró finalmente en el top 20 en 2017, estuvo a punto de hacerlo al situarse en la posición n ${ }^{\circ} 21$. Sin embargo, en 2018 ha entrado con fuerza ocupando un meritorio puesto $\mathrm{n}^{\circ} 8$. Cabe señalar que esta tendencia ha quedado situada muy por delante de la tendencia «tecnología portátil vestible», con la que sin duda se encuentra relacionada, invirtiéndose la tendencia observada en las encuestas internacionales. En las encuestas internacionales la «tecnología portátil vestible» ha sido la tendencia situada en la primera posición en las dos últimas encuestas, colocándose muy por delante de «apps para teléfonos inteligentes». Parecería esperable que en los próximos años se tienda a reproducir progresivamente en las encuestas españolas la pauta que se da en las encuestas internacionales en cuando al orden relativo de ambas tendencias.

Por otro lado, la tendencia relacionada con «clases colectivas» aparece por primera vez en la encuesta de 2018, si bien, en la de 2017 no se consideró como tendencia potencial. Esta tendencia hace referencia a un tipo de actividad que lleva desarrollándose en gimnasios y centros de fitness mucho tiempo y resulta difícil saber cuál ha sido la razón que la ha hecho emerger por primera vez entre las 20 principales tendencias en la encuesta internacional de 2017 y que ese fenómeno se haya reproducido en la encuesta española de 2018. Resultará interesante observar la evolución de esta tendencia en el futuro tanto en el ámbito internacional como en el nacional.

Finalmente, la última tendencia que se incorpora al top 20 este año es la tendencia de «Equipos multidisciplinares de trabajo (médicos, fisioterapeutas, nutricionistas y GCAFYD)». Esto parece mostrar que, en el contexto español, además de profesionales bien formados y cualificados se detecta la necesidad de trabajo multidisciplinar para obtener máximos beneficios y mejores resultados, especialmente en personas que persiguen el objetivo de mejorar ciertos problemas de salud. Esta tendencia puede de algún modo relacionarse con la iniciativa delACSM de «Ejercicio es Medicina ${ }^{\mathbb{}} »$ que propugna la incorporación del ejercicio a las estrategias terapéuticas con las que abordar una amplia variedad de problemas de salud y la derivación de los pacientes a profesionales del ejercicio para encontrar asesoramiento adecuado y desarrollar los programas de ejercicio que más les puedan beneficiar.

\section{Limitaciones y fortalezas}

El presente estudio presenta las limitaciones propias de un estudio basado en encuestas en línea (Díaz de Rada, 2011), es decir, la falta de representatividad poblacional, así como la tasa de respuesta obtenida que, aunque ha sido similar a la obtenida en la encuesta nacional de 2017, es menor a las reportadas en los estudios internacionales del ACSM. El motivo que puede explicar esta menor tasa de respuesta en relación a los estudios internacionales es que en las encuestas llevadas a cabo por el ACSM los encuestados son compensados con un incentivo en especie, que es una de las estrategias documentadas para incrementar la tasa de respuesta en las encuestas online (Sánchez, Muñoz \& Montoro, 2009). Esto, sin embargo, no ha resultado posible en el estudio español por falta de medios para ello.

Por otro lado, la principal fortaleza del estudio es que replica la metodología del ACSM, lo cual permite realizar comparaciones entre los datos nacionales e internacionales. Además, dado que en esta ocasión se realiza el segundo estudio español, resulta posible empezar a trazar patrones de evolución de las tendencias a través del tiempo mediante la comparación con los resultados de la encuesta nacional de 2017. En nuestro conocimiento, estos estudios son los primeros que replican en el ámbito nacional las encuestas del ACSM y permiten obtener información sobre tendencias de fitness en un país específico.

\section{Conclusiones}

A modo de conclusión puede decirse que, en términos generales, las tendencias identificadas en la encuesta nacional para 2018 se corresponden a las identificadas en la encuesta del año 2017, con 17 tendencias coincidentes en el top 20 y tres diferentes. En relación a las tendencias que ocuparon los primeros lugares en la encuesta nacional de 2018 estas ocuparon los seis primeros puestos también en la encuesta nacional del año 2017, mostrando así su preponderancia en la valoración realizada por los profesionales del sector en ambos años. Además, tanto en la encuesta de 2018 como en la de 2017 la primera posición del ranking es ocupada por la tendencia «personal formado, cualificado y experimen- 
tado». Asimismo, las tendencias nacionales para 2018 se corresponden en gran medida con las tendencias internacionales identificadas en la última encuesta de la ACSM disponible (año 2017) con 15 tendencias similares y cinco tendencias diferentes, si bien en este caso solo 3 tendencias coinciden dentro de los seis primeros puestos. Finalmente, resulta destacable que la primera tendencia en ámbito internacional («tecnología portátil vestible») ocupa una discreta posición en la encuesta española de 2018 al situarse en el puesto 16 pese a haber subido dos puestos respecto a la encuesta de 2017.

\section{Referencias}

Cambridge Dictionary (s.f.). Trend. Recuperado de: http:// dictionary.cambridge.org/us/

Díaz de Rada, V. (2011) Ventanas e inconvenientes de la encuesta por Internet. Papers, 97(1), 193-223.

Kohlbacher, F y Hertastt, C. (Eds.) (2008). The Silver Market Phenomenon: Business Opportunities in an Era of Demographic Change, Springer. Berlin.

Reference Dictionary (s.f.) Fad. Recuperado de: http:// dictionary.reference.com/

Sánchez Fernández, J., Muñoz Leiva, F., y Montoro Ríos, F.J (2009) ¿Cómo mejorar la tasa de respuesta en encuestas online? Revista de Estudios Empresariales, 1, 45-62.

Thompson, W.R. (2006). Worldwide survey reveals fitness trends for 2007. ACSM's Heatlh \& Fitness Journal, 10 (6), 8-14.
Thompson, W.R. (2007). Worldwide survey reveals fitness trends for 2008. ACSM's Heatlh \& Fitness Journal, 11 (6), 7-13.

Thompson, W.R. (2008). Worldwide survey reveals fitness trends for 2009. ACSM's Heatlh \& Fitness Journal, 12 (6), 7-14.

Thompson, W.R. (2009). Worldwide survey reveals fitness trends for 2010. ACSM’s Heatlh \& Fitness Journal, 13 (6), 9-16.

Thompson, W.R. (2010). Worldwide survey reveals fitness trends for 2011. ACSM's Heatlh \& Fitness Journal, 14 (6), 8-17.

Thompson, W.R. (2011). Worldwide survey reveals fitness trends for 2012. ACSM’s Heatlh \& Fitness Journal, 15 (6), 9-18.

Thompson, W.R. (2012). Worldwide survey reveals fitness trends for 2013. ACSM's Heatlh \& Fitness Journal, 16 (6), 8-17.

Thompson, W.R. (2013). Now trending: worldwide survey of fitness trend for 2014. ACSM's Heatlh \& Fitness Journal, 17 (7), 10-20.

Thompson, W.R. (2014). Worldwide survey of fitness trend for 2015: what's driving the market. ACSM's Heatlh \& Fitness Journal, 18 (6), 8-17.

Thompson, W.R. (2015). Worldwide survey of fitness trend for $2016.10^{\text {th }}$ anniversary edition. ACSM's Heatlh \& Fitness Journal, 19(6), 9-18.

Thompson, W.R. (2016). Worldwide survey of fitness trend for 2017. ACSM's Heatlh \& Fitness Journal, 20(6), 8-17.

Veiga, O.L., Valcarce Torrente, M., y King Clavero,A. (2017). Encuesta nacional de tendencias en fitness en España para 2017. Apunts. Educación Física y Deportes, 128(2),108-125.

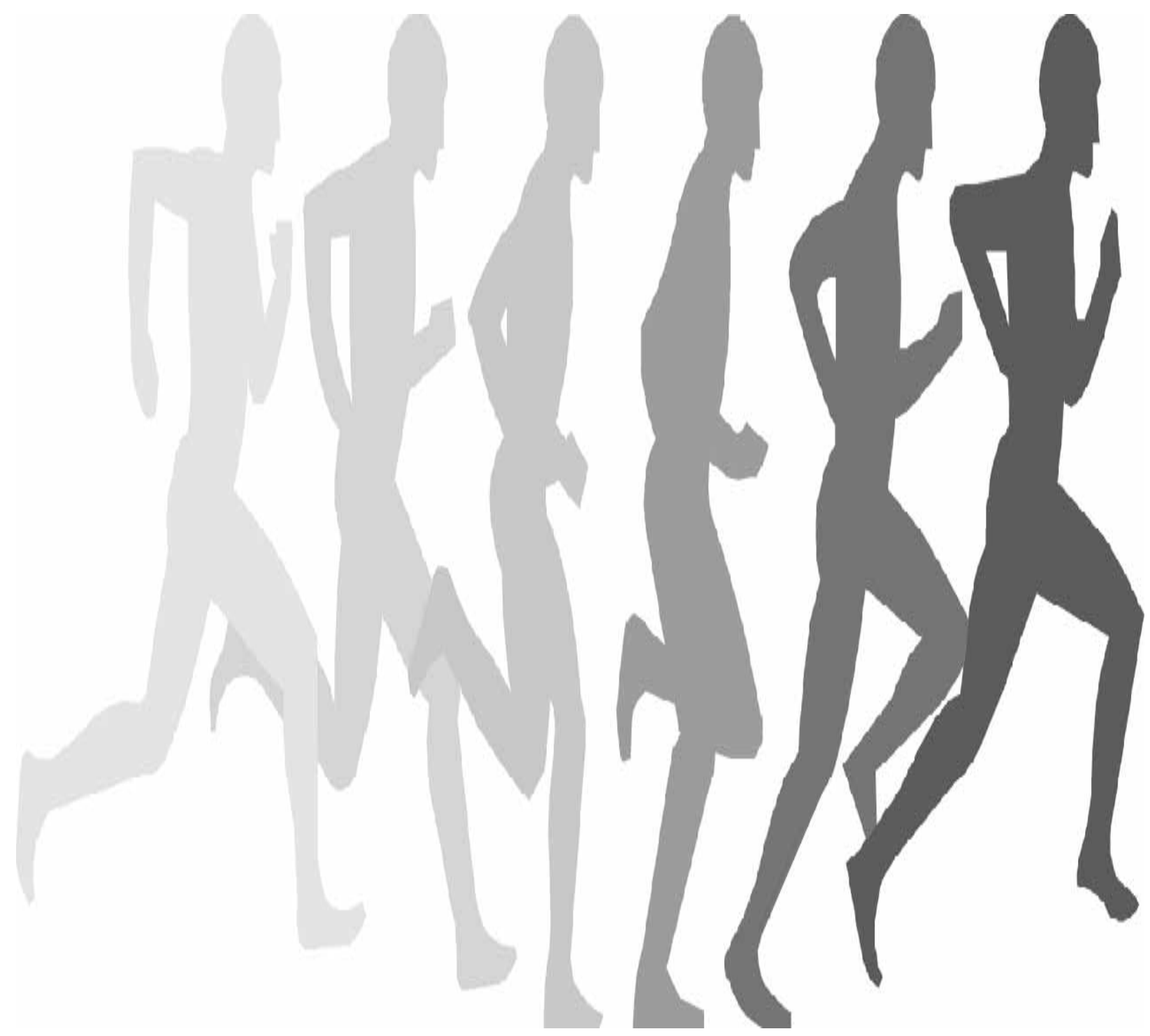

